\title{
Aprendizaje/enseñanza del ritmo inglés en la Educación Primaria y Secundaria
}

\author{
Francisco GutiérRez DíEz \\ Departamento de Filología Inglesa \\ Universidad de Murcia
}

Recibido: 15 mayo 2009/ Aceptado: 15 octubre 2009

ISSN: $1697-7467$

\begin{abstract}
RESUMEN: En este trabajo se presenta una visión general de la problemática de la enseñaza/aprendizaje del ritmo inglés por hispanohablantes y se proponen soluciones fundadas en la investigación empírica de los contrastes rítmicos entre el inglés y el español y en el análisis de errores rítmicos. Se incide en la conveniencia pedagógica de visualizar el ritmo como sustrato necesario en la estructuración de la entonación inglesa. Sobre la base del análisis de los principales contrastes rítmicos entre las dos lenguas y la tipificación de los errores, se ofrecen varias directrices para la didáctica del ritmo. Finalmente se argumenta sobre las ventajas de integrar la problemática de las formas fuertes y débiles del inglés como subsidiaria de la correspondiente al ritmo y la entonación.
\end{abstract}

Palabras clave: prosodia, interlenguaje rítmico, ritmo contrastivo, errores de aprendizaje rítmico, enseñanza del ritmo.

\section{Teaching/Learning English Rhythm in Primary and Secondary Education}

ABSTRACT: In this paper we present an overview of the main problems involved in the teaching/learning of English rhythm to/by Spanish speakers, and propose solutions founded on both contrastive and error analysis. We think that it is pedagogically convenient to visualise rhythm as a necessary stratum in the structuring of English Intonation. On the basis of the analysis of rhythmic contrasts between the two languages and the typification of rhythmic errors, various guidelines for dealing with English rhythm are put forward. We argue over the advantages of integrating the teaching of strong/weak forms of English as ancillary to the teaching of rhythm and intonation.

Key words: Prosody, Rhythmic Interlanguage, Contrastive Rhythm, Rhythmic Errors, the Teaching of Rhythm.

\section{INTRODUCCIÓN}

En una conferencia impartida en la Universidad de Sevilla en 1991, Geoffrey Leech se lamentaba del papel de cenicienta que la gramática ocupaba en la implementación del método comunicativo. Si la suerte de la gramática no parecía boyante en esa época, la de la pronunciación no tenía mejor suerte. Desde entonces la atención a la enseñanza de la pronunciación del componente segmental ha mejorado claramente, aunque la atención prestada a la prosodia 
y, más concretamente, al ritmo es claramente mejorable, tal como se señala en dos tesis doctorales recientes (de Jódar, 2005; Martínez, 2004). El panorama, según las encuestas incluidas en esos dos trabajos, se puede resumir en los siguientes hechos:

a) Por lo que respecta a las vocales y consonantes, se presentan y practican de forma aislada, ignorándose los fenómenos de asimilación, fusión y elisión propios de la cadena hablada, y, sobre todo, el papel determinante del ritmo en la modelación de esos fenómenos segmentales. La fusión y la elisión pueden considerarse como formas extremas de compresión duracional silábica o segmental, o como productos colaterales de dicha compresión, como instrumentos que contribuyen a dar mayor isocronía a los pies rítmicos.

b) Muchos libros de texto utilizados no incluyen en sus materiales la más mínima referencia, siquiera implícita, a los dos componentes prosódicos mencionados. Esa es una de las principales razones por las que el ritmo y la entonación no forman parte de los objetivos didácticos de muchos profesores de Secundaria.

Ante este panorama, resulta prometedor el anuncio del MEC de incorporar una prueba oral de lengua extranjera en las PAU que podrá ser en inglés, francés, alemán, italiano o portugués, a partir del curso 2011/2012. Creemos que esa medida contribuirá a paliar el problema señalado en (b) propiciando la inclusión del ritmo y la entonación inglesa en todos los libros de texto.

A lo largo de esta exposición intentaremos demostrar que el ritmo es una parcela relativamente fácil para los alumnos, pues no se requiere ninguna gradación de los materiales empleados. Además la adquisición del ritmo es facilitadora del aprendizaje de la entonación, por la estrecha relación que existe entre ambos subcomponentes de la prosodia inglesa. De forma análoga, explicaremos por qué el ritmo lingüístico es un marco ideal para la práctica y eventual dominio de las formas fuertes y débiles del inglés, por la contribución de dichas formas a la estructuración de aquél. Nuestra propuesta didáctica, que desgranaremos en el apartado 5, irá precedida de la preceptiva referencia al análisis contrastivo y al análisis de errores (apartados 2-4), con el fin de fundamentar dicha propuesta.

\section{AnÁlisis CONTRASTIVo y ANÁLiSis de ERRORES}

A estas alturas parece una obviedad que los mejores procedimientos para la enseñanza de la lengua extranjera son los que cabe catalogar como comunicativos. Las destrezas orales activa y pasiva son dos caras de la misma moneda: la oralidad del lenguaje hablado/escuchado. Teniendo en cuenta que en cada una de esas destrezas se practican de forma simultánea todos los componentes del lenguaje en sentido amplio, es decir, inclusivos no sólo de la fonología, gramática y vocabulario sino también de la pragmática, es natural que la práctica de la comunicación oral englobe también la práctica de la pronunciación.

Pero la simulación de la comunicación en el aula de lengua extranjera, muy lejos de un entorno nativo, requiere como complemento la realización de ejercicios no sólo gramaticales o de vocabulario sino también los de pronunciación. Lo que está claro es que el aprendizaje lejos del entorno nativo no puede ser igual que el de una lengua nativa. Por si eso fuera poco, 
cabe recordar que en el medio escolar de una lengua nativa también se practica el aprendizaje/ enseñanza de forma explícita para mejorar los usos de la propia lengua. En un entorno no nativo, la misma herramienta - la enseñanza explícita- es parte esencial en el proceso mismo de adquisición lingüística. Este proceso está mediado por la lengua materna del alumno, lo que nos lleva a la necesidad de perfilar los problemas de aprendizaje, entre otros medios, (a) mediante el análisis contrastivo y, como complemento y verificación de las predicciones del mismo, (b) también hay que perfilar los errores de aprendizaje de nuestros alumnos. Sólo a partir de esas dos premisas, y del inventario de problemas de aprendizaje que dichas premisas ayudan a identificar es posible plantearse (c) la formulación de técnicas de enseñanza explícita del ritmo inglés.

El ritmo no es un sistema de términos en contraste lingüístico ya sea al modo de los contrastes fonémicos propios de la fonotaxis segmental o al de los contrastes fonológicos gradados de las distintas configuraciones entonativas. Pero, si bien no tiene una función lingüística, el ritmo de cada lengua tiene una configuración fonética cuya distorsión por el hablante no nativo de esa lengua impide a los hablantes nativos de la misma establecer el interfaz entre el nivel fonético y el fonológico o funcional; dicho de otro modo, la distorsión de la temporización (timing) y la acentuación de las secuencias segmentales del habla impide al oyente traspasar el umbral fonético y percibir los contrastes fonológicos intencionales. De ahí que la temporización correcta de la producción oral del hablante sea condición indispensable para que funcione el correspondiente correlato rítmico-perceptual como marco adecuado para las estructuras lingüísticas bien formadas no sólo en el nivel fonológico sino en el resto de los niveles formales del lenguaje.

Nos gustaría hacer una referencia somera a los paradigmas teóricos sobre los que se ha asentado la descripción teórica del ritmo, y sobre todo su aplicabilidad al análisis contrastivo el análisis de errores y, sobre todo, al aprendizaje/enseñanza del ritmo lingüístico. El paradigma más conocido durante las últimas décadas es el que contempla una tipología fundamentalmente dual del ritmo de las lenguas. Lenguas como el inglés se asignarían al grupo de lenguas acentualmente acompasadas, mientras que el español pertenecería al grupo de las silábicamente acompasadas. En mi opinión, durante mucho tiempo no se ha distinguido claramente entre temporización de las unidades fonético-fonológicas por un lado y el ritmo lingüístico por el otro. La temporización es una propiedad de la producción oral, mientras que el ritmo o ritmicidad lingüística es un atributo perceptual. Los fonetistas han pasado décadas tratando de medir la isocronía física de los intervalos acentuales o de las sílabas de la cadena hablada con la idea de certificar su estatus de correlatos acústicos de las distintas formas de percibir el ritmo. La variabilidad de los datos obtenidos, generalmente alejados de la isocronía de cualquier tipo y la falta generalizada de consenso a la hora de interpretar dichos datos ha llevado a un cierto escepticismo sobre la viabilidad teórica del paradigma que comentamos (véase Grabe y Low, 2002, para una revisión de la literatura sobre el tema).

En el caso de las lenguas isosilábicas, los analistas han relegado, cuando no ignorado, el papel del acento en la configuración del ritmo y han pasado por alto que el isosilabismo por sí solo es antagónico con la idea misma de ritmicidad. A este respecto Gutiérrez (1996: 269) afirma lo siguiente:

El mucho o poco ritmo que haya en la prosa del español se deberá básicmente a la alternancia de sílabas tónicas con sílabas átonas dentro del pie rítmico, siendo el 
timing isosilábico un componente prosódico que, en este caso, actúa como distorsionador rítmico más que como elemento integrante del ritmo.

En inglés, por el contrario, la alternancia entre tónica y átona se da dentro del pie rítmico. En paralelo con esa alternancia se da una temporización de los intervalos interacentuales (i.e. de los pies rítmicos), que si bien no es isócrono en sentido físico, sí está más cerca de la isocronía que el pie rítmico del español, cuya duración está en función del número de sílabas del pie, y está muy alejada de la isocronía acentual del inglés, siendo éste el principal contraste rítmico entre las dos lenguas.

En la última década ha surgido un nuevo paradigma de investigación del ritmo lingüístico. Partiendo del hecho constatado de que es una quimera seguir buscando en la señal acústica del producto de habla la isocronía acentual absoluta de las llamadas lenguas acentualmente acompasadas o el isosilabismo de las referidas como silábicamente acompasadas, el nuevo paradigma trata de establecer la tipología rítmica sobre una nueva base: la variabilidad duracional de los intervalos consonánticos y los vocálicos dentro de la cadena hablada. Los primeros resultados parecen indicar un predominio de la duración media de los intervalos consonánticos sobre la duración media de los intervalos vocálicos en las tradicionalmente denominadas lenguas de isocronía acentual, mientras que la duración de los intervalos vocálicos predominaría sobre la de los intervalos consonánticos en las lenguas isosilábicas (Grabe y Low, 2002; Ramus, Nespor y Mehler, 1999).

En cualquier caso, no percibimos por el momento la posibilidad de trasladar estos hallazgos al aprendizaje o didáctica del ritmo de una lengua extranjera (Gutiérrez, Dellwo, Gavalda y Rosen, 2008). En otras palabras, nadie ha aportado sugerencias sobre posibles estrategias a seguir en el aula de inglés como L2 para convertir en objetivos rítmicos o de simple temporización didácticamente viables la adquisición por los alumnos de la habilidad para producir correctamente los intervalos consonánticos del inglés, más largos que los del español, y los intervalos vocálicos ingleses, más cortos que los del español. En todo caso, se trataría de objetivos derivados de la agrupación fonotáctica de consonantes y vocales en la cadena hablada de cada una de las dos lenguas de forma diferenciada y contrastiva, y en ese sentido se trata de fenómenos segmentales pero no rítmicos. La mayor o menor duración media de los intervalos consonánticos y vocálicos es un subproducto de la agrupación fonotáctica de los segmentos vocálicos y consonánticos (cada uno de los cuales tiene su duración típica).

A falta de pruebas sobre las posibles formas de asociación entre la duración de los intervalos vocálicos y consonánticos y la percepción de la ritmicidad, creemos que siguen vigentes de cara a la aplicabilidad didáctica los conceptos prosódicos tradicionales relacionados con la prominencia y la temporización de la producción oral, como son el pie rítmico y sus partes estructurales (ictus y remiss) y el acento rítmico (también llamado oracional). Por su estrecha relación con factores prosódicos, también son útiles conceptos propios del nivel segmental, como son el debilitamiento vocálico del inglés (determinante de la ausencia de acento) frente a la ausencia del mismo en español, o la compresión duracional de los segmentos prosódicamente determinada en inglés frente a la ausencia de tal compresión en español. 


\section{Análisis contrastivo}

En Gutiérrez y Conde (1990) se argumenta sobre la bondad del pie rítmico de Abercrombie (1967) y Halliday $(1967 ; 1970)$ y sobre otros tipos de unidad rítmica postulados en la literatura, en especial, el grupo rítmico de Allen (1954), Gimson (1970 y 1975) y O’Connor (1967). Además de las ventajas teóricas, entre ellas una mejor comparabilidad del español con el inglés, hay una de tipo didáctico: sólo hay un tipo de pie, el que empieza por una sílaba prominente/tónica/larga y va seguido de un número indeterminado de sílabas no prominentes/ átonas/cortas. Los principales rasgos contrastivos entre ambas lenguas son:

a) Mayor grado de isosilabismo en español que en inglés en la producción oral de ambas lenguas.

b) La razón duracional tónica/átona obtenida para el español es inferior a la del inglés, según diversos estudios. Eso se debe a que la diferencia duracional entre tónica y átona es mucho mayor en inglés que en español. Sobre esa diferencia interlingüística en varios corpora del inglés y el español, véase Gutiérrez (2005).

c) La compresión de la duración silábica dentro de un pie rítmico, a medida que aumenta el número de sílabas del mismo, es mucho mayor en inglés que en español. Este fenómeno fonético se suele asumir como uno de los correlatos fonéticos de la mayor isocronía perceptiva de los pies rítmicos del inglés frente a los del español. La ausencia de compresión silábica significativa en el pie rítmico del español contribuye a reforzar el mayor isosilabismo duracional del español frente al inglés.

d) La compresión silábica del inglés no anula las notables diferencias duracionales entre tónica y átona en esa lengua, muy superiores a las que existen en español. Para un corpus de habla inglesa y otro de habla española, Gutiérrez (2001) halló una ratio de 2.08 para el inglés y 1.52 para el español.

e) Los rasgos apuntados en (a-d) para el inglés son determinantes de un hecho bien demostrado: si bien no se ha podido establecer la isocronía absoluta de los pies rítmicos del inglés, sí hay innumerables estudios cuyos datos avalan una tendencia hacia la isocronía. A modo de ejemplo, en un corpus de habla de Catherine Patch citado por Halliday (1967: 12), la ratio de la duración media de los pies de 1, 2 y 3 sílabas resultó ser 5:6:7, respectivamente.

Existe otro tipo de contraste entre las dos lenguas, que incidirá en el correspondiente planteamiento didáctico que haremos más adelante. Nos referimos a los verbos auxiliares y los pronombres personales que no reciben acento rítmico en inglés, pero sí en español.

\section{Errores de aprendizaje rítmico}

Major (1987) analiza dos tipos de error fonológico, el de interferencia y el evolutivo. El primero está inducido por rasgos presentes en la lengua nativa del alumno y consiste en la transferencia (negativa) de tales rasgos a la lengua meta. Los evolutivos no guardan relación 
con la lengua nativa del aprendiz y son de la misma naturaleza que los errores cometidos cuando se aprende la lengua nativa. Los errores de interferencia no son resultado de los procesos evolutivos, mientras que los errores evolutivos proceden de dichos procesos, que, a su vez, son parte de la gramática universal. Aplicando la dicotomía de Major, hemos detectado los siguientes errores en diversos estudios sobre el aprendizaje rítmico del inglés por alumnos de secundaria:

a) Errores de interferencia:

1. La producción oral de los alumnos suele mostrar una ratio duracional tónica/átona inferior a la del inglés por nativos. Eso se debe en primer lugar a que no alargan las tónicas y/o no acortan las átonas en una medida similar a la de los anglohablantes nativos (Gutiérrez, 2001).

2. Con respecto a la temporización de los pies rítmicos del inglés, los alumnos muestran lo que, a nuestro modo de ver, es el error de interferencia más llamativo y con visos de fosilización: el grado de anisocronía de los pies rítmicos de su inglés es muy similar al del español por nativos y se encuentra a una distancia significativa del grado de isocronía correspondiente al inglés por nativos. Puede hablarse incluso de fosilización. Esta se ha observado no sólo en los alumnos de secundaria sino también en alumnos de nivel avanzado que nunca habían recibido instrucción sobre el ritmo inglés.

3. Los alumnos de inglés no debilitan debidamente las vocales átonas del inglés. La centralización y articulación relajada de las átonas constituye un índice tímbrico que, unido a su acortamiento duracional, contribuye a la percepción de su alternancia rítmica con las vocales tónicas, que son tensas (fuertes), periféricas y más largas. El problema se complica por el diferente tratamiento acentual y rítmico de las palabras «gramaticales» en uno y otro idioma. En español no hay debilitamiento vocálico, ni existe la doble forma fuerte/débil de dichas palabras. Sobre este tema, véase Gutiérrez (2004a y 2004b).

\section{b) Errores evolutivos}

1) En los estadios elementales del aprendizaje, los alumnos utilizan un tempo más lento que el de los nativos ingleses en una tarea de producción oral idéntica. Ese dato apunta a un error evolutivo, que puede tener que ver con el nivel de fluidez del alumno, determinado a su vez por algún déficit de competencia a nivel fonológicosegmental, léxico, gramatical, pragmático, etc.

2) Hay un uso excesivo de pausas y falsos arranques que puede estar motivado por un déficit en la competencia lingüística en comparación con los nativos de la L2 y, posiblemente, por el factor ansiedad.

Varios autores, como Brown (1977), Prator (1971), Taylor (1981) y Wilkins (1975), otorgan mayor trascendencia a este tipo de errores que a los relacionados con los segmentos vocálicos y consonánticos en lo relativo a la comprensión del mensaje. Este se ve distorsionado por el manejo incorrecto de los parámetros del acento y la temporización silábica, lo que contribuye a confundir al interlocutor nativo mediante focalizaciones acentuales erróneas que 
atraen la atención de aquél hacia elementos espurios de la cadena hablada, distrayéndola de otros realmente importantes para la descodificación correcta del mensaje.

\section{Sobre la didÁctica del RitMo}

Dentro de este apartado señalaremos los objetivos concretos relacionados con la didáctica del ritmo (Subapartado 5.1). Seguirá la exposición de algunas claves para la didáctica del ritmo (Subapartado 5.2). Se trata de una reflexión sobre la filosofía pedagógica aplicable a las técnicas y procedimientos a utilizar. Seguirá una referencia al tratamiento de las formas fuertes y débiles, con una doble propuesta: integrar la enseñanza de las formas débiles en la del ritmo, y la de las formas fuertes en la de la entonación (Subapartado 5.3). Finalizaremos con una crítica de un modelo de didáctica del ritmo que ha sido muy popular en las últimas décadas y cuyos puntos débiles son una concepción de la unidad rítmica teóricamente obsoleta y una aplicación pedagógica poco eficaz derivada de aquella (Subapartado 5.4).

Lo primero que queremos señalar a propósito del título del presente trabajo, es que, dada la enseñanza reglada del inglés a lo largo de los distintos niveles oficiales, creemos que la enseñanza del ritmo se debe acometer en la enseñanza primaria y secundaria ya que la primera se nutre de principiantes y la segunda de falsos principiantes o de los niveles preintermedio e intermedio, siendo esos niveles cruciales para la adquisición del ritmo por varias razones:

1) Porque es un componente relativamente fácil de enseñar y aprender. Cualquier material didáctico se presta a ese fin, no siendo necesaria en este caso la gradación de materiales que puede ser exigible para otros componentes lingüísticos en virtud del nivel de suficiencia del alumno. Cualquier material pluriverbal que sirva para la realización de tareas comunicativas es apto para la enseñanza del ritmo.

2) La ausencia o postergación del tratamiento del ritmo a niveles superiores de aprendizaje, puede no ser suficiente para erradicar la fosilización de errores rítmicos inducidos en niveles inferiores en muchos casos por la ausencia de ese componente en el aula de inglés como L2. Anteriormente hemos aludido a la anisocronía acentual del inglés de alumnos hispanohablantes de nivel avanzado (Gutiérrez, 2001) como error de fosilización.

3) La enseñanza del ritmo es facilitadora de la enseñanza de un aspecto de la entonación conocido tradicionalmente como tonicidad (Halliday, 1967). Ésta es un subsistema entonativo consistente en la focalización informativa dentro de la unidad entonativa o unidad tonal. Dicha focalización se realiza mediante la acentuación tonal o entonativa de los dos puntos estructurales más importantes de la unidad entonativa, como son el núcleo entonativo (i.e. sílaba inicial de la palabra con mayor prominencia entonativa de la unidad tonal) y la sílaba de arranque (onset), i.e. la primera sílaba con acento tonal de la unidad tonal. Como es sabido, el núcleo inicia la configuración tonal final portadora de los contrastes significativos primarios de la unidad tonal. La sílaba de arranque inicia el segmento prenuclear (o cabeza) que puede ser portador de funciones entonativas secundarias. Como se mencionó anteriormente, el ritmo interviene en la estructuración de la entonación, y ahora podemos completar esa afirmación diciendo que, para que una sílaba pueda recibir acento tonal ya sea como núcleo o como 
sílaba de arranque de la unidad entonativa, tiene que haber recibido previamente un acento rítmico. Concluyendo, la enseñanza del ritmo puede no sólo facilitar la enseñanza de la entonación sino también evitar que los alumnos cometan errores de interferencia consistentes en la asignación errónea del núcleo y la sílaba de arranque a palabras que normalmente $y$, salvo que medien razones de énfasis o contraste informativo, no pueden recibir acento rítmico.

\subsection{Objetivos para la didáctica del ritmo}

A continuación presentamos y explicamos los ingredientes rítmicos que, a nuestro juicio, constituyen objetivos de enseñanza:

a) Alternancia rítmica: este primer ingrediente resulta de la mezcla serial de sílabas tónicas con sílabas átonas dentro de la cadena hablada. El hecho de que la alternancia rítmica se apoye directamente en la distribución de los acentos rítmicos apunta a la necesidad de practicar también la acentuación correcta de las palabras inglesas (acento léxico).

b) Debilitamiento vocálico: el debilitamiento de las sílabas átonas está estrechamente ligado al tema de la alternancia rítmica. En este sentido me gustaría llamar la atención sobre un tipo de alternancia rítmica al que Bolinger (1981) se ha referido con el nombre de «ritmo silábico»: se trata de la alternancia entre dos tipos de cualidad vocálica: la de las vocales «plenas» (full) con las «reducidas» (reduced). La verdad es que los términos plena y reducida son polivalentes: plena significaría tónica, periférica y larga, y reducida significaría átona, central (o centralizada) y corta. De esa forma, además de los contrastes tónica/átona y larga/corta se destacaría el contraste rítmico entre los rasgos periférico y central. Los contrastes larga/corta y periférica/central, no se dan a nivel fonémico en español, lo que supone un handicap para los alumnos hispanohablantes de ILE y una posible fuente de interferencia. Al mismo tiempo, el hecho de que los contrastes tónica/átona y periférica/centralizada del inglés se den de forma simultánea sobre las mismas secuencias de segmentos, contribuye a incrementar el grado de ritmicidad del inglés en comparación con el español.

c) Temporización de sílabas y pies rítmicos: este es otro de los elementos a tener en cuenta en la planificación docente. Para empezar, las sílabas tónicas del inglés son mucho más largas que las átonas. Recordemos en este punto la ratio representativa de la diferencia promedio entre uno y otro tipo de sílabas para un corpus analizado en Gutiérrez (2001): 2.08 para el inglés y 1.52 para el español.

d) Isocronía acentual: relacionado con la temporización de sílabas y pies está el tema de la isocronía acentual, es decir, la duración similar de los intervalos entre acentos como algo específico del inglés frente al español. Éste es un aspecto muy polémico que merece un comentario. Se ha establecido por numerosos estudios la inexistencia de la isocronía absoluta en el sentido cronológico de la palabra, incluso en los casos en que las condiciones experimentales (series de pies rítmicos en que estos contenían el mismo número de sílabas) se diseñaron con la intención de conseguirla, como es el caso de O'Connor (1968). Tal resultado, no es de extrañar, ya que el mecanismo articulatorio está constreñido por una serie de limitaciones de tipo neuromuscular, que 
impiden el logro, no sólo de la isocronía interacentual, sino la repetición del mismo sonido en términos físico-acústicos. En todo caso, la falta de isocronía absoluta a nivel de producción no debe preocuparnos, puesto que el ritmo pertenece al mecanismo perceptivo del oyente que lo superpone a la señal acústica que oye. Lo más probable es que baste con la tendencia a la isocronía (lo que Halliday, 1967 denomina «isocronía fonológica») en la señal acústica (en lugar de la isocronía absoluta) para que el oyente superponga el ritmo a la mencionada tendencia.

d) Compresión silábica: en inglés la percepción isócrona o casi isócrona tiene por índice el efecto de la compresión silábica, es decir el acortamiento duracional del pie rítmico a medida que aumenta el número de sílabas del mismo. Ese es un fenómeno recurrente en muchos autores y sólo puede explicarse como factor coadyuvante de lo que en el párrafo anterior llamábamos tendencia a la isocronía interacentual En los pies polisilábicos, la compresión silábica parece afectar más a las sílabas átonas que a las tónicas.

e) Regla de duración silábica extra y regla de préstamo duracional: Bolinger (1981) señala, en relación con la temporización de sílabas y pies rítmicos, lo siguiente: cuando una sílaba larga va seguida de otra larga, la primera recibe una duración extra. Cuando una sílaba larga va seguida de una corta, la duración de la primera se acorta en beneficio de la segunda. Si utilizamos el símbolo L para las sílabas largas, L+ para las extralargas y C para las cortas, el siguiente ejemplo de Bolinger servirá de ilustración.

$$
\begin{aligned}
& \text { Quite / possibly / no / real / happiness can be / found in } \\
& \mathrm{L}+\mathrm{L} \mathrm{C} \mathrm{C}+\mathrm{L}+\mathrm{L} \mathrm{C} \mathrm{C} \mathrm{C} \mathrm{C} \mathrm{C} \\
& \text { / all / these / struggles and / all / this / fan / fare } \\
& \mathrm{L}+\mathrm{L}+\mathrm{L} \mathrm{C} \mathrm{C}+\mathrm{L}+\mathrm{L}+\mathrm{L}+
\end{aligned}
$$

Las sílabas quite, no, real, all, these, all, this y fan- van seguidas de otra larga; la sílaba -fare va seguida de pausa. Por consiguiente, todas ellas son extralargas, es decir, L+

\subsection{Inducción y explicitación de las reglas rítmicas}

Antes de nada, debe insistirse en la necesidad de ayudar al alumno a reconocer los patrones rítmicos antes de su producción. En ese sentido, los ejercicios de reconocimiento de segmentos textuales alternarán con los de producción de dichos segmentos. Es necesaria la referencia a los patrones rítmicos dentro del proceso didáctico. Aunque eso pueda sonar a método explícito-deductivo, no lo es, ya que los patrones no se introducen para que el alumno deduzca a partir de esos patrones o reglas los correspondientes usos individuales, sino a modo de referente, síntesis, o recapitulación de lo ya practicado por procedimientos propios de una aproximación comunicativa al aprendizaje de la pronunciación. Para que así sea, partimos del supuesto de que la presentación de esos patrones debe ir totalmente desprovista de ropaje teórico-descriptivo para no caer en los vicios del viejo método «gramática-traducción».

La formulación pedagógica de los patrones rítmicos deberá ser asequible para los alumnos. Para la práctica del ritmo, los patrones no tienen por qué formularse como reglas explí- 
citas y completas, sino que puede bastar con hacer referencia a las manifestaciones de esas reglas en la mayoría de los textos que se emplean en el aula de inglés. Los ejemplos (2-5), que ofrecemos a continuación, nos servirán para ilustrar lo que venimos diciendo:

(2) «En cada una de las palabras o frases que vais a oír, todas las sílabas menos la

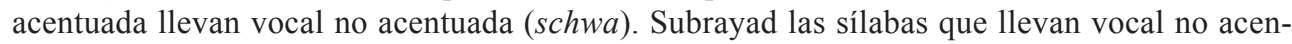
tuada.»

tailor, survival, however, protect him, with the doctor ...

(3) «En cada una de las oraciones que vais a oír, todas las sílabas menos las acentuada llevan vocal no acentuada. Subrayad las sílabas que no se acentúan».

Simon was the author of the pamphlet

(4) «En el texto que tenéis delante y que acabáis de escuchar, la duración de los tramos entre las sílabas acentuadas (en negrita) es similar. Repetid el texto marcando con un golpe en el pupitre cada acento. La duración entre golpes sucesivos debe ser siempre la misma».

\section{The race was about to start. Carl was on lane number three ...}

(5) «Cuando una sílaba acentuada va seguida de otra acentuada o de una pausa, la primera de ellas es más larga de lo normal. Escuchad el texto anterior [ejemplo (4)] y subrayad las sílabas que reciben duración extra». [En el ejemplo anterior la respuesta sería start, lane y three].

En los cuatro enunciados anteriores, lo que se le señala al alumno son eventos de la estructura superficial, seguidos de una instrucción relativa a una tarea (de comprensión auditiva en (2), (3) y (5), y de lectura en voz alta en (4). Tales eventos están determinados por una serie de reglas rítmicas de tipo subyacente, y por ende abstracto, que no necesitamos enseñar de forma explícita al alumno. Es más, la misma tarea apuntada en cada uno de los cuatro enunciados puede servir directa o indirectamente para practicar el dominio de más de una regla subyacente, sin cambiar siquiera los ejemplos utilizados.

Mediante el ejercicio (2), además de la acentuación léxica, se pueden practicar la alternancia tónica/átona, la alternancia plena/débil y la alternancia vocal central/vocal periférica. Mediante el (3), se practican las mismas reglas que en (2), sólo que ahora se hace en contextos más amplios (las oraciones), y el alumno comienza a estar expuesto, a nivel de percepción, al ensamblaje de pies rítmicos en dichos contextos. Mediante el ejercicio (4), además de incidir en las reglas practicadas en (2-3), se añade la práctica de otra regla: la regla de la isocronía entre pies rítmicos. Mediante el ejercicio (5), además de las reglas mencionadas hasta el momento, se introduce la práctica de las reglas de compresión silábica, préstamo duracional y duración silábica extra.

A la luz de los ejemplos (2-5), puede advertirse lo que decíamos más arriba: la enseñanza del ritmo puede y debe enmarcarse fundamentalmente en una aproximación metodológica de tipo explícito-inductivo, en que el alumno induce las reglas a partir de la solución de tareas. 
En cualquier caso, la mención de las reglas rítmicas debe ser esporádica y a modo de corolario que sigue a su práctica e inducción por parte del alumno.

Es tarea del profesor asegurarse de que todas las reglas rítmicas del inglés, que él sí debe conocer de forma analítica y expresa, tienen un lugar en el correspondiente programa didáctico; y que cualquier referencia a ellas, sea de forma parcial o de forma completa, debe hacerse prescindiendo de la jerga técnica propia de la lingüística teórica, y utilizando en su lugar una un lenguaje pedagógico.

Queremos resaltar que, desde el punto de vista analítico, las reglas de compresión silábica, préstamo duracional y duración silábica extra son más complejas que la regla de alternancia rítmica y la regla de isocronía acentual (o duración casi isócrona de los pies rítmicos). Sin embargo, desde el punto de vista pedagógico, eso no supone una mayor dificultad, puesto que al practicar la isocronía de los pies rítmicos, estamos practicando de forma sintética o integrada la compresión silábica, el préstamo duracional y la duración silábica extra, que son las que en su conjunto posibilitan la isocronía de los pies rítmicos. En otras palabras, ni siquiera hay necesidad de explicar al alumno estas tres reglas, y sería suficiente, sobre todo en los primeros niveles, con explicarles la alternancia rítmica y la duración casi isócrona de los pies (por supuesto, en los momentos en que proceda dentro del guión práctica-explicitación al que nos venimos refiriendo). Eso sí, el profesor debe conocer las cinco reglas y, según el nivel del alumno o su dificultad para construir la isocronía acentual, puede serle de ayuda la explicitación y práctica por separado de esas tres reglas subsidiarias de la isocronía acentual.

Afortunadamente, la dificultad que existe para compatibilizar una gradación de las estructuras gramaticales con el criterio de función comunicativa, es inexistente en el caso que a nosotros nos ocupa: el ordenamiento priorizado de los componentes rítmicos como objetivos didácticos se puede llevar a la práctica con cualquier material lingüístico, cualquier función comunicativa y cualquier tarea docente/discente relacionada con las destrezas audio-orales. Dicho de otra manera, cualquier material, cualquier lección del programa sirve para la práctica del ritmo inglés. Este pequeño detalle junto con otros de mayor calibre expuestos más arriba debería hacer reflexionar a los que aún sostienen que la enseñanza explícita de la pronunciación casa mal con la aproximación comunicativa al aprendizaje de una lengua extranjera.

En principio el alumno debería estar expuesto desde el primer momento a todos los componentes rítmicos a nivel de percepción. A nivel de producción, la práctica puede ser globalizada y parcial, es decir, centrándose alternativamente en uno u otro componente rítmico a lo largo del programa didáctico.

Por globalizada ha de entenderse la práctica consistente en la escansión rítmica hecha por el alumno a base de marcar los intervalos interacentuales de un texto. La práctica parcial o diferenciada se centraría, por este orden, en las reglas de alternancia rítmica, préstamo duracional y duración silábica extra. La gradación que se propone debe ser complementada con una presentación cíclica de los distintos componentes rítmicos. Nuestra hipótesis es que la enseñanza del ritmo en los estadios elemental y preintermedio de aprendizaje propiciando la práctica globalizada/parcial de sus componentes haría innecesaria la enseñanza parcial a partir del nivel preintermedio, siendo suficiente para ése y el resto de niveles superiores la práctica globalizada (es decir, la escansión rítmica para consolidar la isocronía acentual) de forma esporádica. A esta hipótesis subyace nuestra opinión de que es doblemente lamentable el que no se enseñe el ritmo en los primeros estadios del aprendizaje; primero por ser un componente fonético esencial para la construcción del nivel fonológico representado por la entonación y, 
segundo, porque su aprendizaje en los primeros estadios, además de impedir la fosilización de errores rítmicos, convertiría en redundante su presencia como objetivo didáctico en los niveles superiores del aprendizaje, cosa que no ocurre con el resto de componentes fonológicos segmentales o prosódicos ni con el sintáctico, discursivo y pragmático, con los que hay que seguir bregando en los niveles superiores.

\subsection{Sobre la didáctica de las formas fuertes y débiles}

Nos gustaría retomar este tema desde una perspectiva poco destacada hasta el momento: el papel subsidiario de las formas fuertes y débiles en relación con el ritmo y con el subsistema entonativo denominado tonicidad. Comenzaremos por avanzar nuestra hipótesis al respecto: la didáctica de las formas débiles debe integrarse en la enseñanza del ritmo y la de las formas fuertes, en la didáctica de la tonicidad. La enseñanza de las formas débiles también facilita de forma indirecta la enseñanza de la tonicidad. De esa forma, además de practicar el ritmo, se facilitará a los alumnos el aprendizaje de la tonicidad por la vía de impedir que focalicen las formas débiles mediante la acentuación tonal errónea.

La presentación de las formas débiles debe hacerse con perspectiva contrastiva, es decir, se debe incidir en la práctica de la no acentuación rítmica de los verbos auxiliares, los pronombres personales y algunas preposiciones monosilábicas (en contraste con el comportamiento acentual de estas formas en español).

La enseñanza de las formas débiles (palabras función) no tiene por que ser una tarea independiente y separada, sino integrada en la enseñanza y en la práctica del ritmo. Dada la gran frecuencia de las palabras no acentuables desde el primer día de clase, el planteamiento didáctico es simple: no es precisa la selección de materiales especiales, sino que sirven los mismos que se utilizan para cualquier otro tipo de tarea discente/docente. Al mismo tiempo que se van practicando las reglas rítmicas aludidas más arriba, se le van corrigiendo in situ al alumno los errores de acentuación de formas débiles, utilizando como feedback una referencia explícita a la categoría de palabra que no admite acentuación rítmica y que ha sido objeto de error. En los ejemplos (5-6) aparecen formas débiles de las palabras aludidas:

(6) «En cada una de las palabras o frases que vais a oír, todas las sílabas menos las acentuada llevan vocal débil. Subrayad las sílabas que llevan vocal débil.»

Jack was shocked at the news you gave him.

(7) «En cada una de las oraciones que vais a oír, todas las sílabas menos la acentuada llevan vocal débil. Subrayad las sílabas que llevan vocal débil».

They read the notice carefuly

En estos dos ejemplos, las respectivas instrucciones incluyen la expresión «vocal débil», en lugar de «vocal no acentuada», que es lo que aparecía en las instrucciones de los ejemplos (2-3) en el apartado 5.1. Por fortuna, resulta intuitivamente sencillo hacer comprender a los alumnos que la vocal débil, por el mero hecho de serlo, no se acentúa, o a la inversa, no lleva acento por ser débil (sin necesidad de explicarles el hecho fonético del relajamiento articulatorio 
de la mayor parte de las vocales centrales o las vocales centralizadas, que resultaría más difícil de entender por el alumno).

Tampoco es necesario enseñar de forma explícita las palabras función cuya conducta acentual sea idéntica en inglés y español, como es el caso de las conjunciones y determinantes posesivos. Aunque los hispanohablantes también cometen errores consistentes en la acentuación rítmica de tales palabras en el nivel elemental de aprendizaje, se trata de errores evolutivos que desaparecerán relativamente pronto de la interlengua de los alumnos a partir del nivel intermedio. Los que sí corren peligro de fosilizarse si no se corrigen y contrarrestan con la práctica sistemática del ritmo son los errores consistentes en la acentuación rítmica de los pronombres personales, los verbos auxiliares y las preposiciones del inglés (es decir, los errores de interferencia). En Gutiérrez (2008), se ofrece un inventario de errores de alumnos hispanohablantes de inglés consistentes en la asignación del núcleo o sílaba de arranque a palabras que no admiten acentuación rítmica y por ende tampoco pueden recibir acentuación tonal.

Por lo que respecta a la enseñanza de las formas fuertes de aquellas palabras que pueden tener formas débiles (las tres categorías morfológicas antes aludidas), es un tema que pertenece a la didáctica específica de la tonicidad. Se trataría de enseñar la selección correcta de las sílabas/palabras candidatas a convertirse en núcleo o sílaba de arranque mediante la correspondiente acentuación tonal. En el ejemplo (8) se ilustra la selección de la forma fuerte de la preposición on como núcleo entonativo de la unidad tonal correspondiente por razones de contraste.

(8) // I said "!ON the desk // ('not under the desk')

Los términos de la focalización y la forma de realizarla pertenecen al ámbito específico de la enseñanza de la entonación, pero ese es otro tema que escapa al objetivo general del presente trabajo.

\subsection{La inadecuación de la aproximación didáctica basada en el «grupo rítmico»}

Nos referiremos ahora a otro tema polémico relacionado con la enseñanza del ritmo. Se trata de la utilización del denominado grupo rítmico (rhythmic group) como unidad rítmica. Esta unidad no es, en principio, adecuada por consistir sólo en palabras o frases; la palabra y la frase son constituyentes gramaticales. Los tipos de secuencia tónica/átona que caben en la unidad frase son indefinidos en cuanto a número y morfología debido a la complejidad estructural de la misma (premodificación, postmodificación, complementación, etc.) potenciada a su vez por la variabilidad fonológica de las palabras que la integran. Elevar la palabra y/ o las frases del inglés a la categoría de unidades rítmicas es teóricamente insostenible y es una temeridad extrapolar tales unidades al ámbito pedagógico, por dos razones:

a) La primera, de tipo teórico, es que el pie rítmico es una unidad fonético-fonológica, no una unidad gramatical. Si la estructura de los constituyentes gramaticales determinase la estructura de los pies rítmicos, sería incontrolable el número de tipos de pies rítmicos posibles. 
b) Ese número indefinido e incontrolable de tipos de pie rítmico resultaría poco aplicable al aprendizaje/enseñanza de un ritmo tan variopinta y complejamente estructurado.

En las ofertas pedagógicas que asumen esa variabilidad del denominado grupo rítmico, se representan los distintos tipos de grupo rítmico como secuencias de rayas y puntos (ChelaFlores, 1988) o de cuadraditos grandes y pequeños (Allen, 1967) para representar respectivamente las sílabas tónicas/largas y las átonas/breves. La consecuencia inmediata es que el alumno se desanima de entrada ante tamaña variabilidad y renuncia a controlarla. Queremos subrayar que nuestra crítica no se centra en el valor simbólico de las rayas/puntos o cuadrados grandes/pequeños como símbolos para representar las sílabas tónicas y átonas, sino en la complejidad de configuraciones generadas por las secuencias de esos símbolos en los materiales didácticos adscritos a la corriente del grupo rítmico. De hecho, esos símbolos son tan viables como los de cualquier otra simbología dicotómica (i.e. representativa de sílabas tónicas y átonas), como es el caso de la representación de las sílabas tónicas en cursiva o en negrita y de las átonas en letra normal.

En un estudio empírico de Gutiérrez y Conde (1990) se pone de manifiesto la ventaja de la utilización del pie rítmico (rhythmic foot) de Halliday (1967 y 1970) en lugar del grupo rítmico. Dicha ventaja se fundamenta en las siguientes consideraciones:

a) El pie rítmico se concibe como una unidad fonológica cuyos límites pueden coincidir o no con límites de constituyentes inmediatos. Se trata de un único tipo de pie rítmico simple y con un gran poder predictivo. Otra ventaja teórica es que propicia una mayor economía y elegancia a la hora de describir el ritmo como sistema prosódico asociado al sistema prosódico de la entonación: las secuencias rítmicas serían constituyentes de la unidad entonativa, tal como afirma Halliday. Ya hemos aludido más arriba a las ventajas de esa asociación de cara a la enseñanza de la entonación.

b) La mayor simplicidad del pie rítmico lo convierte en más atractivo para su aplicación a la enseñanza/aprendizaje del ritmo, puesto que la memoria del alumno no se ve sobrecargada por la presentación e imposible retención de la ilimitada tipología del grupo rítmico.

Además de la mayor adecuación teórica del pie rítmico, está su mayor eficacia pedagógica, ya que puede utilizarse con cualquier tipo de secuencia comunicativa —oración, párrafo y texto - desde el primer día de clase para la práctica de la alternancia tónica/átona, la isocronía interacentual, la compresión silábica, el préstamo duracional y la duración extra. Las palabras aisladas pueden servir para la práctica subsidiaria del ritmo sólo en un sentido: la práctica del acento léxico, en especial cuando está gramaticalmente determinado (éxport) expórt, súbject/subjéct, cónduct/condúct, etc.).

\section{REFERENCIAS}

Abercrombie, D. (1967). Elements of General Phonetics. Edinburgh: Edinburgh Unversity Press. Allen, S.W. (1970). Living English Speech. Stress and Intonation Practice for the Foreign Student. London: Longman. 
Bolinger, D. (1981). Two kinds of vowels, two kinds of rhythm. Bloomington: Indiana University Linguistics Club.

Brown, G. (1977). Listening to Spoken English. London: Longman.

Chela-Flores, B. (1998). Teaching English Rhythm. Caracas: Fondo editorial Tropycos.

Grabe, E. \& Low E.L. (2002). «Durational variability in speech and the rhythm class hypothesis». En C. Gussenhoven y N. Warner (eds.), Papers in Laboratoratory Phonology, 7: 515-546. Berlin: Mouton de Gruyter.

Gutiérrez, F. (2008). «Contrastive intonation and error analysis. Tonality and tonicity in the language of a group of Spanish learners of English». En M.A. Gómez, J.L. Mackenzie y E.M. Ganzález (eds.), Languages and Cultures in Contrast and Comparison. Amsterdam: John Benjamins, 327-354.

Gutiérrez, F. (2004a). «Acento y debilitamiento vocálico en el inglés de un grupo de estudiantes españoles de Enseñanza Secundaria Obligatoria (ESO)». Porta Linguarum, 2: 111-124.

Gutiérrez, F. (2004b). «Contraste entre las vocales átonas del inglés y el español. El debilitamiento vocálico en el inglés de un grupo de hispanohablantes». En E. González y A. Rollings (eds.) Studies in Contrastive Linguistics. Santiago: Publicaciones de la Universidad de Santiago de Compostela, 211-222.

Gutiérrez, F. (2005). «Aprendizaje de la pronunciación del español por anglohablantes. Distorsión rítmica y timing». En C. Butler, M.A. Gómez González y S. Doval (eds.), The Dynamics of Language Use. Amsterdam: John Benjamins, pp. 287-306.

Gutiérrez, F. (2001). The acquisition of English syllable timing by native Spanish speakers learners of English. En empirical study. En R. Monroy and F. Gutiérrez (eds), Perspectives on Interlanguage Phonetics and Phonology. International Journal of English Studies, 1, 1: 93-114.

Gutiérrez, F. (1996). «Aprendizaje de la pronunciación del español por anglohablantes. Distorsión rítmica y timing.» En F. Gutiérrez (ed.), El español lengua internacional (I Congreso Internacional de AESLA, Universidad de Granada). Murcia: Compobell, pp. 267-276.

Gutiérrez, F. y Conde, C. (1990). «Supuestos dudosos en la enseñanza del ritmo inglés a hispanohablantes». En F. Garrudo y F. Comesaña (eds.), Actas del VII Congreso Nacional de Lingüistica Aplicada (Universidad de Sevilla), 265-273.

Gutierrez, F., Dellwo, V., Gavalda, N. y Rosen, S. (2008). «The development of L2 speech rhythm in Spanish learners of English (A)». (Resumen de la comunicación presentada al Meeting of the Acoustical Society of America, 2008). Journal of the Acoustical Society of America, 123: p. 3886.

Gimson, A.C. (1970). Introduction to the Pronunciation of English. London: Edward Arnold.

Gimson, A.C. (1975). A Practical Course of English Pronunciation. London: Edward Arnold.

Halliday, M.A.K. (1967). Intonation and Grammar in British English. The Hague: Mouton.

Halliday, M.A.K. (1970). A Course in Spoken English. Intonation. Oxford: Oxford University Presss.

Jódar de, O. (2005). Estudio sobre los efectos de un tratamiento alternativo del componente fonológico en el currículo de lengua inglesa para $2^{\circ}$ de Bachillerato (Tesis doctoral defendida en la Universidad de Murcia).

Major, R. (1987). «A model for interlanguage phonology». En G. Ioup y S. Weinberger (eds.), Interlanguage Phonology. Cambridge, Mass: Newbury House, 121-124.

Martínez. F. (2004). Estudio de una intervención pedagógica para la enseñanza de la pronunciación inglesa en $4^{\circ}$ curso de la ESO (Tesis doctoral defendida en la Universidad de Murcia). 
O’Connor, J.D. (1967). Better English Pronunciation. Cambridge: Cambridge University Press. Prator, C.H., (1971). «Phonetics vs. phonemics in the ESL classroom: when is allophonic accuracy important?». TESOL Quarterly, 5, 1: 61-72.

Ramus, F., Nespor, M. y Mehler, J. (1999). «Correlates of linguistic rhythm in the speech signal». Cognition, 73: 265-292.

Taylor, D.S. (1981). «Non-native speakers and the rhythm of English». International Review of Applied Linguistics, 19: 219-26.

Wilkins, S.A. (1975). Linguistics in Language Teaching. London: Edward Arnold. 